\title{
A Simple and Environmentally Benign Protocol for Biginelli Reactions Catalyzed by Silica-Bonded $S$-Sulfonic Acid
}

\author{
Mahmood TAJBAKHSH ${ }^{1, *}$, Yousef RANJBAR ${ }^{2}$, Abdolhosein MASUODI $^{2}$, Samad KHAKSAR $^{3}$ \\ ${ }^{1}$ Chemistry Department, University of Mazandaran, PO Box 453, Babolsar, Iran \\ ${ }^{2}$ Chemistry Department, Payame Noor University, PO Box 4697, Tehran, Iran \\ ${ }^{3}$ Chemistry Department, Islamic Azad University, Ayatollah Amoli Branch, PO Box 678, Amol, Iran
}

\begin{abstract}
A protocol for the synthesis of 3,4-dihydropyrimidin-2(1H)-ones and -thiones was developed by means of a three-component condensation of an aldehyde, a $\beta$-dicarbonyl compound, and urea or thiourea in acetic acid catalyzed by silica-bonded $S$-sulfonic acid. Compared to the classical Biginelli reaction conditions, this new protocol has the advantages of consistently excellent yields and short reaction times. After the reaction, the catalyst could be recovered easily and reused with little change in its activity.
\end{abstract}

Key words: Biginelli reaction; silica-bonded $S$-sulfonic acid; dihydropyrimidinone

CLC number: O643 Document code: A

Received 20 February 2012. Accepted 11 July 2012.

*Corresponding author.Tel: +98-1125234338; Fax:+98-1125242002; E-mail: tajbaksh@umz.ac.ir

This work was supported by the Research Council of Payame Noor University.

English edition available online at Elsevier ScienceDirect (http://www.sciencedirect.com/science/journal/18722067).

Organic syntheses in which heterogeneous acid catalysts [1] are used in place of homogeneous acid reagents have received much attention owing to their potential for use in the development of green [2] and sustainable chemical processes [3]. Reactions catalyzed by solid acids have the potential to be safe and highly atom efficient, and they typically involve simple workup procedures that do not produce large amounts of salt wastes [4]. In recent decades, dihydropyrimidinones and their derivatives have drawn attention because of their antibacterial, antiviral, antitumor, and anti-inflammatory activities [5]. For example, Biginelli compounds (3,4-dihydropyrimidin-2(1H)-ones) act as calcium channel blockers, antihypertensive agents, a 1A-antagonists, and neuropeptide $\mathrm{Y}$ antagonists [6-9]. Batzelladine B, an alkaloid isolated from a marine sponge, contains a dihydropyrimidine unit and shows anti-HIV activity [10]. Numerous procedures for preparing dihydropyrimidinones have been reported, involving reagents and catalysts such as $\mathrm{H}_{4} \mathrm{PMo}_{11} \mathrm{VO}_{40}$ [11], Dowex-50W [12], $\mathrm{H}_{3} \mathrm{PW}_{12} \mathrm{O}_{40} / \mathrm{SiO}_{2}$ [13], $\mathrm{MgBr}_{2}$ [14], polymer-supported 4-aminoformoyldiphenylammonium triflate [15], Na$\mathrm{HSO}_{4} / \mathrm{SiO}_{2}$ [16], $\mathrm{FeCl}_{3}$ [17], $\mathrm{ZrCl}_{4}$ [18], $\mathrm{Cu}(\mathrm{OTf})_{2}$ [19],
$\mathrm{Bi}(\mathrm{OTf})_{3}$ [20], ytterbium triflate [21], $\mathrm{NH}_{2} \mathrm{SO}_{3} \mathrm{H}$ [22], 12-molybdophosphoric acid [23], natural HEU-type zeolites [24], $\mathrm{Sr}(\mathrm{OTf})_{2}$ [25], $\mathrm{ZrOCl}_{2} \cdot 8 \mathrm{H}_{2} \mathrm{O}$ [26], silica triflate [27], $\mathrm{Fe}\left(\mathrm{HSO}_{4}\right)_{3}$ [28], TCICA [29], $\mathrm{PPh}_{3}$ [30], melamine trisulfonic acid [31], $\mathrm{Fe}_{3} \mathrm{O}_{4}$ nanoparticles [32], sulfonated $\beta$-cyclodextrine [33], metal hydrogen sulfates [34], ionic liquids [35-37], and sulfonated carbon materials [38].

However, some of these procedures require expensive reagents, strongly acidic conditions, long reaction times, high temperatures, or stoichiometric amounts of catalysts, or they result in environmental pollution or give unsatisfactory yields. Therefore, there is a need for new catalysts that are readily available or easy to prepare, inexpensive, and recoverable (hence reusable and environmentally friendly). Moreover, the workup procedure should be simple. In this regard, functionalized silica particles and mesoporous silica materials have been synthesized and have found many applications [39-43]. As part of our continuing studies of organic processes mediated by heterogeneous acid catalysts, we now report a highly efficient synthesis of 3,4-dihydropyrimidin-2(1H)-ones and -thiones (Scheme 1) by means of a three-component condensation reaction catalyzed by sil-

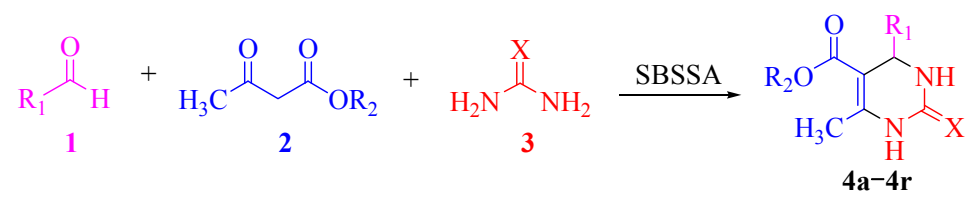

Scheme 1. SBSSA-catalyzed synthesis of 3,4-dihydropyrimidin- 2(1H)-ones and -thiones. 
ica-bonded $S$-sulfonic acid (SBSSA).

\section{Experimental}

SBSSA was prepared according to Refs. [44,45]. In a general procedure, a mixture of an aldehyde $(2 \mathrm{mmol})$, a $\beta$-dicarbonyl compound ( $2 \mathrm{mmol})$, urea or thiourea (3 $\mathrm{mmol})$, glacial acetic acid $(5 \mathrm{ml})$, and $\operatorname{SBSSA}(0.05 \mathrm{~g})$ was heated with stirring at $110{ }^{\circ} \mathrm{C}$ for $1 \mathrm{~h}$. After completion of the reaction (monitored by thin-layer chromatography), the catalyst was removed by filtration, and the filtrate was poured into ice water $(20 \mathrm{ml})$. The resulting solid precipitate was filtered and recrystallized from ethanol to give pure 3,4-dihydripyrimidin-2(1H)-one or -thione. Products were identified by comparison of their ${ }^{1} \mathrm{H}$ and ${ }^{13} \mathrm{C}$ NMR spectra and melting points with those of authentic samples. Spectroscopic data for selected products are listed as follows.

Methyl 1,2,3,4-tetrahydro-6-methyl-2-oxo-4-phenylpyrimidine-5-carboxylate (4a). m.p. $228-230{ }^{\circ} \mathrm{C}$; ${ }^{1} \mathrm{H}$ NMR $(300$ MHz, DMSO-d ${ }_{6}$ ): $\delta 9.16$ (br s, $1 \mathrm{H}, \mathrm{NH}$ ), 7.78 (br s, $1 \mathrm{H}$, $\mathrm{NH}), 7.22-7.36(\mathrm{~m}, 5 \mathrm{H}), 5.25(\mathrm{~d}, 1 \mathrm{H}, J=2.4 \mathrm{~Hz}), 2.24$ (s, 3H), 2.07 (s, 3H); ${ }^{13} \mathrm{C}$ NMR: $\delta 194.4,158.5,152.1,147.8$, 136.9, 127.7, 113.9, 109.6, 55.1, 53.3, 30.18, 18.8 .

Methyl 1,2,3,4-tetrahydro-4-(4-methoxyphenyl)-6-methyl2-oxopyrimidine-5-carboxylate (4b). m.p. $165-167{ }^{\circ} \mathrm{C} ;{ }^{1} \mathrm{H}$ NMR (300 MHz, DMSO-d d $) \delta 9.16$ (br s, $1 \mathrm{H}, \mathrm{NH}), 7.78$ (br s, $1 \mathrm{H}, \mathrm{NH}), 7.16(\mathrm{~d}, 2 \mathrm{H}, J=8.7 \mathrm{~Hz}), 6.88(\mathrm{~d}, 2 \mathrm{H}, J=$ $8.7 \mathrm{~Hz}), 5.20(\mathrm{~d}, 1 \mathrm{H}, J=3.0 \mathrm{~Hz}), 3.72(\mathrm{~s}, 3 \mathrm{H}), 2.28(\mathrm{~s}, 3 \mathrm{H})$, 2.07 (s, 3H); ${ }^{13} \mathrm{C}$ NMR: $\delta$ 194.4, 158.5, 152.1, 147.8, 136.4, $127.7,113.9,109.6,55.1,53.3,30.2,18.8$.

Methyl 1,2,3,4-tetrahydro-6-methyl-4-(4-nitrophenyl)-2oxopyrimidine-5-carboxylate (4c). m.p. $226-228{ }^{\circ} \mathrm{C} ;{ }^{1} \mathrm{H}$ NMR (300 MHz, DMSO-d d $): \delta .40$ (br s, $1 \mathrm{H}, \mathrm{NH}$ ), 8.21 $(\mathrm{d}, 2 \mathrm{H}, J=8.4 \mathrm{~Hz}), 7.93$ (s, 1H), $7.51(\mathrm{~d}, 2 \mathrm{H}, J=8.7 \mathrm{~Hz})$, 5.39 (s, 1H), 2.32 (s, 3H), 2.19 (s, 3H); ${ }^{13} \mathrm{C}$ NMR: $\delta$ 194.0, 152.0, 151.6, 149.1, 146.7, 127.7, 123.8, 109.5, 53.1, 30.7, 19.1.

Ethyl 1,2,3,4-tetrahydro-4-isopropyl-6-methyl-2-oxopyrimidine-5-carboxylate (4j). m.p. $192-194{ }^{\circ} \mathrm{C} ;{ }^{1} \mathrm{H}$ NMR $(300$ MHz, DMSO-d d $_{6}: \delta 8.86$ (br s, $1 \mathrm{H}, \mathrm{NH}$ ), 7.26 (br s, $1 \mathrm{H}$, $\mathrm{NH}), 4.04(\mathrm{~m}, 2 \mathrm{H}), 3.96(\mathrm{t}, 1 \mathrm{H}, J=3.6 \mathrm{~Hz}), 2.18(\mathrm{~s}, 3 \mathrm{H})$, $1.68(\mathrm{~m}, 1 \mathrm{H}), 1.19(\mathrm{t}, 3 \mathrm{H}, J=7.1 \mathrm{~Hz}), 0.82(\mathrm{~d}, 3 \mathrm{H}, J=6.9$ $\mathrm{Hz}), 0.74(\mathrm{~d}, 3 \mathrm{H}, J=6.8 \mathrm{~Hz}) ;{ }^{13} \mathrm{C}$ NMR: $\delta$ 165.8, 153.2, $148.4,98.2,59.1,55.5,34.6,18.5,17.7,16.0,14.2$.

\section{Results and discussion}

To optimize the reaction conditions, we used benzaldehyde, ethyl acetoacetate, and urea as substrates under various reaction conditions both in the absence and in the presence of SBSSA (Table 1). In the absence of catalyst, the reaction failed to give the desired product at room tempera-
Table 1 Effects of SBSSA amount, reaction temperature, and solvent on product yield

\begin{tabular}{rccccc}
\hline Entry & $\begin{array}{c}\text { SBSSA } \\
\text { amount }(\mathrm{g})\end{array}$ & $\begin{array}{c}\text { Temperature } \\
\left({ }^{\circ} \mathrm{C}\right)\end{array}$ & Solvent & $\begin{array}{c}\text { Time } \\
(\mathrm{h})\end{array}$ & $\begin{array}{c}\text { Yield } \\
(\%)\end{array}$ \\
\hline 1 & 0 & r.t. & HOAc & 24 & 0 \\
2 & 0 & 110 & HOAc & 10 & 60 \\
3 & 0.05 & 110 & HOAc & 1 & 81 \\
4 & 0.10 & 110 & HOAc & 3 & 81 \\
5 & 0.01 & 110 & HOAc & 2 & 65 \\
6 & 0.05 & 100 & - & 2 & 70 \\
7 & 0.05 & reflux & $\mathrm{EtOH}^{2}$ & 5 & 60 \\
8 & 0.05 & reflux & $\mathrm{CH}_{2} \mathrm{Cl}$ & 10 & 15 \\
9 & 0.05 & reflux & $\mathrm{H}_{2} \mathrm{O}$ & 12 & 20 \\
10 & 0.05 & reflux & $\mathrm{CH}_{3} \mathrm{CN}$ & 6 & 60 \\
\hline
\end{tabular}

Reaction conditions: benzaldehyde $2 \mathrm{mmol}$, ethyl acetoacetate 2 mmol, urea $3 \mathrm{mmol}$, solvent $5 \mathrm{ml}$.

ture, even after prolonged reaction time ( $24 \mathrm{~h}$, entry 1$)$. Increasing the temperature to $110{ }^{\circ} \mathrm{C}$ resulted in only a $10 \%$ yield of the desired product (entry 2 ) in the absence of catalyst. However, when $0.05 \mathrm{~g}$ of catalyst was added, the yield increased to $81 \%$ after a reaction time of only $1 \mathrm{~h}$ (entry 3 ). Increasing the amount of catalyst and the reaction time did not improve the yield further (entry 4), and decreasing the amount of catalyst and the reaction time reduced the yield (entry 5). The reaction was also carried out in various other solvents $\left(\mathrm{H}_{2} \mathrm{O}, \mathrm{CH}_{3} \mathrm{CN}, \mathrm{CH}_{2} \mathrm{Cl}_{2}\right.$, and $\left.\mathrm{EtOH}\right)$ and under solvent-free conditions. Under these conditions, the reactions were sluggish, and the formation of byproducts was observed (entries 6-10).

To evaluate the substrate scope of the reaction, we evaluated a variety of substituted aromatic aldehydes and alkyl acetoacetate esters under the optimal reaction conditions, and we obtained good to excellent yields of the corresponding 3,4-dihydropyrimidin-2(1H)-ones and -thiones (Table 2). Both electron-withdrawing and electron-donating substituents on the aromatic ring of the aldehydes gave high yields of the desired products (entries 1-9, 12-14). Interestingly, an acid-sensitive carbon-carbon double was not affected by the reaction conditions, and a high yield of the desired product was obtained (entry 11). However, an aliphatic aldehyde gave a relatively low yield (entry 10 ). The reaction also proceeded smoothly with thiourea and various substituted aldehydes to afford 3,4-dihydropyrimidin-2(1H)thiones (entries 15-18).

To further explore the substrate scope of the reaction, we replaced ethyl acetoacetate with 1,3-cyclohexanedione or dimedone. As expected, these cyclic substrates also underwent smooth, one-pot conversion to give the corresponding dihydropyrimidinones in good yields (Scheme 2). In all cases, the pure product was isolated by simple filtration, without the need for chromatography or a cumbersome workup. 
Table 2 SBSSA-catalyzed synthesis of 3,4-dihydropyrimidin-2(1H)-ones and -thiones

\begin{tabular}{|c|c|c|c|c|c|c|c|}
\hline \multirow{2}{*}{ Entry } & \multirow{2}{*}{$\mathrm{R}^{1}$} & \multirow{2}{*}{$\mathrm{R}^{2}$} & \multirow{2}{*}{$\mathrm{X}$} & \multirow{2}{*}{ Product } & \multirow{2}{*}{ Yield* (\%) } & \multicolumn{2}{|c|}{ Melting point $\left({ }^{\circ} \mathrm{C}\right)$} \\
\hline & & & & & & Found & Reported \\
\hline 1 & $\mathrm{C}_{6} \mathrm{H}_{5}$ & $\mathrm{CH}_{3}$ & $\mathrm{O}$ & $4 a$ & 78 & $228-230$ & $223-236[31]$ \\
\hline 2 & $4-\mathrm{MeO}-\mathrm{C}_{6} \mathrm{H}_{4}$ & $\mathrm{CH}_{3}$ & $\mathrm{O}$ & $4 b$ & 80 & $165-167$ & $168-170[31]$ \\
\hline 3 & $4-\mathrm{O}_{2} \mathrm{~N}-\mathrm{C}_{6} \mathrm{H}_{4}$ & $\mathrm{CH}_{3}$ & $\mathrm{O}$ & $4 c$ & 91 & $226-228$ & $228-230[31]$ \\
\hline 4 & $\mathrm{C}_{6} \mathrm{H}_{5}$ & $\mathrm{OC}_{2} \mathrm{H}_{5}$ & $\mathrm{O}$ & $4 d$ & 81 & $200-202$ & $201-203[32]$ \\
\hline 5 & $4-\mathrm{O}_{2} \mathrm{~N}-\mathrm{C}_{6} \mathrm{H}_{4}$ & $\mathrm{OC}_{2} \mathrm{H}_{5}$ & $\mathrm{O}$ & $4 e$ & 93 & 205-207 & $207-210[31]$ \\
\hline 6 & $4-\mathrm{MeO}-\mathrm{C}_{6} \mathrm{H}_{4}$ & $\mathrm{OC}_{2} \mathrm{H}_{5}$ & $\mathrm{O}$ & $4 f$ & 89 & 196-198 & $199-201[31]$ \\
\hline 7 & 4-Cl- $\mathrm{C}_{6} \mathrm{H}_{4}$ & $\mathrm{OC}_{2} \mathrm{H}_{5}$ & $\mathrm{O}$ & $4 g$ & 88 & $210-212$ & $210-212[31]$ \\
\hline 8 & $3-\mathrm{Cl}-\mathrm{C}_{6} \mathrm{H}_{4}$ & $\mathrm{OC}_{2} \mathrm{H}_{5}$ & $\mathrm{O}$ & $4 h$ & 92 & 190-192 & $193-195[32]$ \\
\hline 9 & $4-\mathrm{Me}-\mathrm{C}_{6} \mathrm{H}_{4}$ & $\mathrm{OC}_{2} \mathrm{H}_{5}$ & $\mathrm{O}$ & $4 i$ & 89 & $212-214$ & $215-216[31]$ \\
\hline 10 & $\left(\mathrm{CH}_{3}\right)_{2} \mathrm{CH}$ & $\mathrm{OC}_{2} \mathrm{H}_{5}$ & $\mathrm{O}$ & $4 j$ & 43 & $192-194$ & $194-195[31]$ \\
\hline 11 & $\mathrm{PhCH}=\mathrm{CH}_{2}$ & $\mathrm{OC}_{2} \mathrm{H}_{5}$ & $\mathrm{O}$ & $4 k$ & 86 & $223-226$ & $222-224[32]$ \\
\hline 12 & 4-HO- $\mathrm{C}_{6} \mathrm{H}_{4}$ & $\mathrm{OC}_{2} \mathrm{H}_{5}$ & $\mathrm{O}$ & 41 & 74 & $203-205$ & $202-204[31]$ \\
\hline 13 & $3-\mathrm{Br}-\mathrm{C}_{6} \mathrm{H}_{4}$ & $\mathrm{OC}_{2} \mathrm{H}_{5}$ & $\mathrm{O}$ & $4 m$ & 85 & $188-190$ & $187-189[32]$ \\
\hline 14 & $2-\mathrm{O}_{2} \mathrm{~N}-\mathrm{C}_{6} \mathrm{H}_{4}$ & $\mathrm{OC}_{2} \mathrm{H}_{5}$ & $\mathrm{O}$ & $4 n$ & 71 & $279-281$ & $187-189[32]$ \\
\hline 15 & $\mathrm{C}_{6} \mathrm{H}_{5}$ & $\mathrm{OC}_{2} \mathrm{H}_{5}$ & $\mathrm{~S}$ & 40 & 86 & 205-207 & $208-210[33$ \\
\hline 16 & $4-\mathrm{MeO}-\mathrm{C}_{6} \mathrm{H}_{4}$ & $\mathrm{OC}_{2} \mathrm{H}_{5}$ & $\mathrm{~S}$ & $4 p$ & 74 & $146-148$ & $150-152$ \\
\hline 17 & $4-\mathrm{Me}-\mathrm{C}_{6} \mathrm{H}_{4}$ & $\mathrm{OC}_{2} \mathrm{H}_{5}$ & $\mathrm{~S}$ & $4 q$ & 85 & 190-192 & $192-194[33$ \\
\hline 18 & 4-Cl- $\mathrm{C}_{6} \mathrm{H}_{4}$ & $\mathrm{OC}_{2} \mathrm{H}_{5}$ & $\mathrm{~S}$ & $4 r$ & 71 & 193-195 & $192-194[33$ \\
\hline
\end{tabular}

Reaction conditions: aldehyde $2 \mathrm{mmol}, \beta$-dicarbonyl compound $2 \mathrm{mmol}$, urea or thiourea $3 \mathrm{mmol}$, glacial acetic acid $5 \mathrm{ml}, \mathrm{SBSSA} 0.05 \mathrm{~g}, 110{ }^{\circ} \mathrm{C}, 1$ h. "Isolated yield.

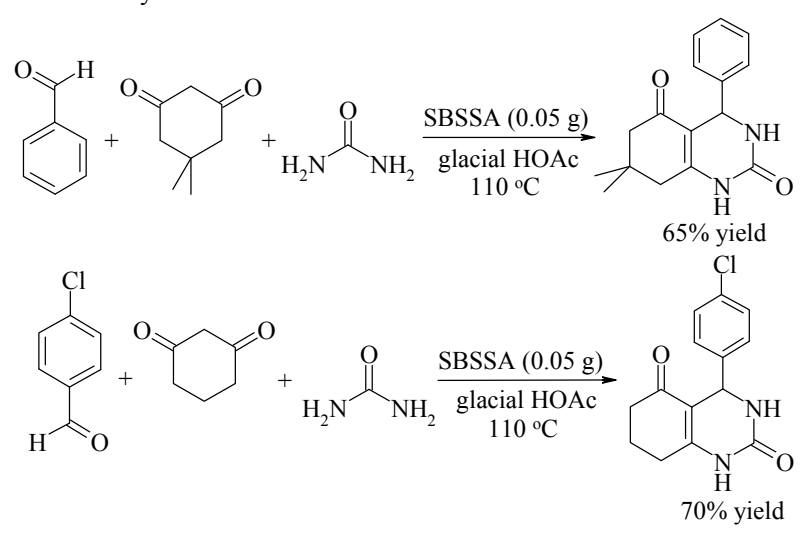

Scheme 2. SBSSA-catalyzed condensations of aldehydes, cyclic 1,3-dicarbonyl compounds, and urea.

To explore the advantages and drawbacks of this SBSSA-catalyzed reaction, we compared the results we obtained under the optimized conditions with results reported in the literature for Biginelli reactions mediated by other catalysts (Table 3 ). The yield of the SBSSA-catalyzed reaction was comparable to or higher than the yields obtained with all but one of the other catalysts listed in Table 3. However, all the other catalysts required longer reaction times. According to the mechanism suggested by Yamamoto [50], we have a Brønsted acid-assisted Brønsted acid catalyst catalytic system (HOAc + SBSSA), and this catalyst system showed higher activity than the other catalysts listed in Table 3.

We also evaluated the feasibility of reusing the SBSSA catalyst. We allowed benzaldehyde, ethyl acetoacetate, and urea to react under the optimized reaction conditions and
Table 3 Comparison of the SBSSA-catalyzed reaction with Biginelli reactions mediated by other catalysts

\begin{tabular}{lcccc}
\hline Catalyst & $\begin{array}{r}\text { Temperature } \\
\left({ }^{\circ} \mathrm{C}\right)\end{array}$ & $\begin{array}{c}\text { Time } \\
(\mathrm{h})\end{array}$ & $\begin{array}{c}\text { Yield } \\
(\%)\end{array}$ & Ref. \\
\hline $\mathrm{HZSM}-5$ & 100 & 12 & 21 & {$[46]$} \\
$\mathrm{HY}$ & 100 & 12 & 80 & {$[46]$} \\
Montmorillonite KSF & 100 & 10 & 35 & {$[47]$} \\
$\mathrm{HCl}^{*}$ & 78 & 18 & 75 & - \\
$\mathrm{Natural} \mathrm{HEU} \mathrm{type} \mathrm{zeolite}^{*}$ & 100 & $4-5$ & 75 & {$[48]$} \\
$\mathrm{SiO}_{2}-\mathrm{R}-\mathrm{SO}_{3} \mathrm{H}$ & 80 & 8 & 90 & {$[49]$} \\
$\mathrm{SBSSA}^{*}$ Classical Biginelli conditions. & 110 & 1 & 81 & this work \\
\hline
\end{tabular}

then filtered the reaction mixture, washed the collected solid catalyst with hot $\mathrm{EtOH}$, dried it in air, and reused it in a second reaction with the same substrates. We were able to reuse the SBSSA catalyst six times without any appreciable loss in its catalytic activity (Fig. 1).

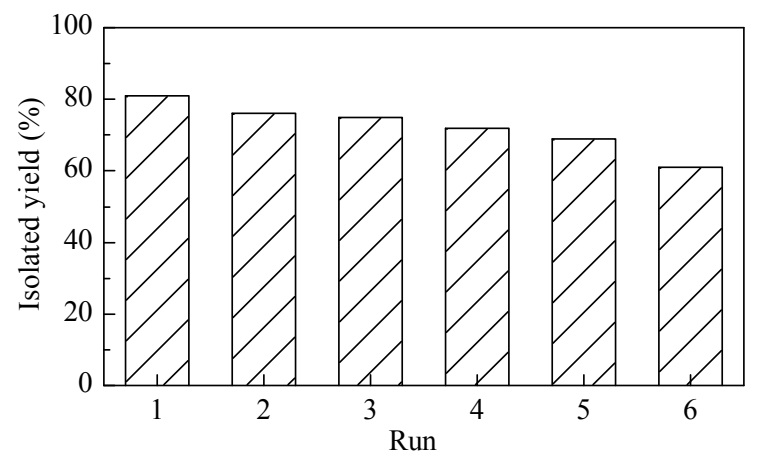

Fig. 1. Recyclability of SBSSA for one-pot synthesis of dihydropyrimidinones. 


\section{Conclusions}

We developed a simple, new procedure for the one-pot synthesis of 3,4-dihydropyrimidine-2(1H)-ones and -thiones by means of a three-component condensation catalyzed by SBSSA in acetic acid at $110^{\circ} \mathrm{C}$. This method offers several advantages: the catalyst is recyclable and inexpensive, the procedure is environmentally friendly, the reaction time is short, the yields are high, and the workup and isolation are easy.

\section{References}

1 Trost B M. Science, 1991, 254: 1471

2 Sheldon R A. Chemtech, 1994, 24: 38

3 Anastas P T, Warner J C. Green Chemistry: Theory and Practice. Oxford: Oxford Press, 1998

4 Kaneda K. Synlett, 2007: 999

5 Kappe C O. Tetrahedron, 1993, 49: 6937

6 Atwal K S, Rovnyak G C, O’Reilly B C, Schwartz J. J Org Chem, 1989, 54: 5898

7 Atwal K S, Swanson B N, Unger S E, Floyd D M, Moreland S, Hedberg A, O’Reilly B C. J Med Chem, 1991, 34: 806

8 Rovnyak G C, Atwal K S, Hedberg A, Kimball S D, Moreland S, Gougoutas J Z, O'Reilly, B C, Schwartz J, Malley M F. J Med Chem, 1992, 35: 3254

9 Kappe C O, Fabian W M F. Tetrahedron, 1997, 53: 2803

10 Patil A D, Kumar N V, Kokke W C, Bean M F, Freyer A J, De Brosse C, Mai S, Truneh A, Carte B. J Org Chem, 1995, 60: 1182

11 Maradur S P, Gokavi G S. Catal Commun, 2007, 8: 279

12 Singh K, Arora D, Singh S. Tetrahedron Lett, 2006, 47: 4205

13 Rafiee E, Shahbazi F. J Mol Catal A, 2006, 250: 57

14 Salehi H, Guo Q X. Synth Commun, 2004, 34: 171

15 Lei M, Wu D D, Wei H G, Wang Y-G. Synth Commun, 2009, 39: 475

16 Chari M A, Syamasundar K. J Mol Catal A, 2004, 221: 137

17 Lu J, Bai Y. Synthesis, 2002: 466

18 Reddy C V, Mahesh M, Raju P V K, Babu T R, Reddy V V N. Tetrahedron Lett, 2002, 43: 2657

19 Prabhakar A S, Dewkar G K, Sudalai A. Tetrahedron Lett, 2003, 44: 3305

20 Varala R, Alam M M, Adapa S R. Synlett, 2003, 67

21 Ma Y, Qian C T, Wang L M, Yang M. J Org Chem, 2000, 65: 3864

22 Kotharkar S A, Jadhav M R, Nagawade R R, Bahekar S S, Shinde D B. Lett Org Chem, 2005, 2: 662

23 Heravi M M, Bakhtiari K, Bamoharram F F. Catal Commun, 2006, 7: 373
24 Tajbakhsh M, Mohajerani B, Heravi M M, Ahmadia A N. J Mol Catal A, 2005, 236: 216

25 Su W K, Li J J, Zheng Z G, Shen Y C. Tetrahedron Lett, 2005, 46: 6037

26 Shirini F, Zolfigol M A, Mollarazi E. Synth Commun, 2006, 36: 2307

27 Shirini F, Marjani K, Nahzomi H T. Arkivoc, 2007, (1): 51

28 Shirini F, Zolfigol M A, Abri A R. J Iran Chem Soc, 2008, 5: 96

29 Shirini F, Zolfigol M A, Mollarazi E. Lett Org Chem, 2005, 2 718

30 Debache A, Amimour M, Belfaitah A, Rhouati S, Carboni B. Tetrahedron Lett, 2008, 49: 6119

31 Shirini F, Zolfigol M A, Albadi J. Chin Chem Lett, 2011, 22: 318

32 Nasr-Esfahani M, Hoseini S J, Mohammadi F. Chin J Catal, 2011, 32: 1484

33 Asgharia S, Tajbakhsh M, Kenari B J, Khaksar S. Chin Chem Lett, 2011, 22: 127

34 Niknam K, Zolfigol M A, Hossieninejad Z, Daneshvar N. Chin J Catal, 2007, 28: 591

35 Peng J J, Deng Y Q. Tetrahedron Lett, 2001, 42: 5917

36 Li M, Guo W S, Wen L R, Li Y F, Yang H Z. J Mol Catal A, 2006, 258:133

37 Zheng R W, Wang X X, Xu H, Du J X. Synth Commun, 2006, 36: 1503

38 Moghadas M, Davoodnia A, Heravi M, Tavakoli-Hoseini N. Chin J Catal, 2012, 33: 706

39 Chong A S M, Zhao X S. Catal Today, 2004, 293: 93

40 Zareyee D, Asghari R, Khalilzadeh M. Chin J Catal, 2011, 32 1864

41 Kang T, Park Y G, Yi J H. Ind Eng Chem Res, 2004 43: 1478

42 Rac B, Molnar A, Forgo P, Mohai M, Bertoti I. J Mol Catal A, 2005, 244: 46

43 Rac B, Hegyes P, Forgo P, Molnar A. Appl Catal A, 2006299 : 193

44 Niknam K, Saberi D, Sefat M N. Tetrahedron Lett, 2009, 50: 4058

45 Niknam K, Mohammadizadeh M R, Mirzaee S. Chin J Chem, 2011, 29: 1417

46 Radha Rani V, Srinivas N, Radhakishan M, Kulkarni S J, Raghaan K V. Green Chem, 2001, 3: 305

47 Bigi F, Carloni S, Frullanti B, Maggi R, Sartori G. Tetrahedron Lett, 1999, 40: 3465

48 Tajbakhsh M, Mohajerani B, Heravi M, Ahmadi A. J Mol Catal A, 2005, 236: 216

49 Gupta R, Paul S, Gupta R. J Mol Catal A, 2007, 266: 50

50 Yamamoto H, Futatsugi K. Angew Chem, Int Ed, 2005, 44: 1924 